Revue d'histoire de l'Amérique française

REVUE D.HISTOIRE DE L'AMÉRIQUE FRANÇAISE

\title{
Artilleurs canadiens-français dans la bataille de Normandie (juillet-août 1944)
}

\section{Jacques Gouin}

Volume 16, numéro 2, septembre 1962

URI : https://id.erudit.org/iderudit/302197ar

DOI : https://doi.org/10.7202/302197ar

Aller au sommaire du numéro

Éditeur(s)

Institut d'histoire de l'Amérique française

ISSN

0035-2357 (imprimé)

1492-1383 (numérique)

Découvrir la revue

Citer cet article

Gouin, J. (1962). Artilleurs canadiens-français dans la bataille de Normandie (juillet-août 1944). Revue d'histoire de l'Amérique française, 16(2), 240-253. https://doi.org/10.7202/302197ar d'utilisation que vous pouvez consulter en ligne.

https://apropos.erudit.org/fr/usagers/politique-dutilisation/ 


\title{
ARTILLEURS CANADIENS-FRANÇAIS DANS LA BATAILLE DE NORMANDIE (juillet-août 1944)
}

\author{
DE CAEN À FALAISE AVEC LE 4e RÉGIMENT \\ CANADIEN D'ARTILLERIE MOYENNE *
}

1er article

\section{INTRODUCTION}

Ce n'est qu'au cours de la seconde guerre mondiale que, pour la première fois dans l'histoire du Canada, depuis l'époque de Frontenac tout au moins, les Canadiens français purent enfin se distinguer dans ce domaine de l'art militaire qu'est l'artillerie.

En effet, ce deuxième conflit mondial favorisa la création au Canada des unités d'artillerie canadiennes-françaises suivantes : $20^{\mathrm{e}}$ et $22^{\mathrm{e}}$ régiments de campagne ; $3^{\mathrm{e}}, 4^{\mathrm{e}}, 12^{\mathrm{e}}, 17^{\mathrm{e}}, 41^{\mathrm{e}}, 52^{\mathrm{e}}$, $60^{\circ}, 61^{\circ}$ et $63^{\mathrm{e}}$ batteries lourdes antiaériennes; $57^{\mathrm{e}}$ batterie légère antiaérienne; $59^{\mathrm{e}}$ batterie côtière; $29^{\mathrm{e}}$ troupe antiaérienne; $82^{\circ}$ batterie du $4^{\mathrm{e}}$ régiment antichars; et enfin le $4^{\mathrm{e}}$ Régiment d'artillerie moyenne.

De toutes ces unités d'artillerie de langue française, seul le $4^{\mathrm{e}}$ Régiment, en tant qu'unité homogène, a participé sans interruption à une campagne complète, celle du nord-ouest de l'Europe, de juillet 1944 à mai 1945. Si la $82^{\mathrm{e}}$ batterie du $4^{\mathrm{e}}$ régiment antichars a ouvert le feu pour la première fois, en Italie, le 16 janvier 1944, soit six mois environ avant le $4^{\text {e }}$ Régi-

* Série d'articles extraits d'un ouvrage en préparation, intitulé: Par la bouche de nos canons: Histoire du $4^{e}$ Régiment canadien d'artillerie moyenne (1941-1945) (à paraître vers 1965). 
ment, à l'été de 1944 en Normandie, il reste que ce dernier a été le seul régiment canadien d'artillerie de langue française, complet dans tous ses cadres, qui ait pourchassé l'ennemi commun jusqu'à la victoire finale en Allemagne, le 6 mai $1945^{1}$.

Du point de vue du Canada français, il nous a semblé que l'histoire de ce régiment valait la peine d'être racontée; que ses exploits, tant en France, en Belgique, en Hollande, qu'en Allemagne, méritaient de figurer dans les annales héroïques de notre race. Nous avons pensé qu'ainsi nous pourrions peut-être effacer jusqu'à un certain point ce sentiment d'humiliation que suscite l'affirmation de Guy Frégault dans son ouvrage intitulé La Guerre de la conquête, à propos de l'attitude des Canadiens français après 1760: «Après avoir vécu sous un gouvernement de type militaire, avoir fourni des capitaines et des combattants à toute la Nouvelle-France et même à la métropole et s'être fait une réputation de «belliqueux », on verra ce groupe humain, et à plus d'une reprise, unanime sur un seul point, lui toujours si divisé: le refus de porter les armes. ${ }^{2}$ »

Nous espérons démontrer, par le récit qui va suivre, que, deux siècles après avoir été «brisé en tant que peuple », les Canadiens français furent encore capables de fournir «des capitaines et des combattants » à leur pays, voire de refaire leur réputation de «belliqueux ».

\section{ORIGINES DU REGIMENT}

Mobilisé dans la province de Québec à l'été de 1941 , le $4^{\circ}$ Régiment d'artillerie moyenne devait connaître bien des mésaventures avant de devenir l'unité d'élite qu'il fut. Pour assumer le commandement de cette nouvelle unité d'artillerie canadiennefrançaise, constituée d'abord en régiment de campagne de trois batteries, un militaire de carrière, le lieut.-col. L.-H.-M. de Bellefeuille-Panet, était rappelé du Royaume-Uni au Canada. Les trois commandants de batteries étaient, au début: le major J.-H.-

1 Renseignements obtenus du lieut-col. C. P. Stacey, alors qu'il était directeur de la Section historique de l'Armée, au ministère de la Défense nationale, Ottawa, lettre du 7 août 1958.

2 (Montréal et Paris, Fides, 1955), 456. 
Réal Gagnon ${ }^{2 a}$, le major Maurice Archer ${ }^{2 b}$, tous deux diplômés du Collège Militaire Royal de Kingston, et le major M. Hallé. Celui-ci ne devait pas servir, en définitive, avec le $4^{\mathrm{e}}$ Régiment, vu que ce dernier, devenu unité d'artillerie moyenne, ne comporta plus désormais que deux batteries au lieu des trois que comportait un régiment de campagne.

Après des mois de difficultés de toutes sortes, le Régiment, qui essayait péniblement de se constituer, recevait du quartier général de la Défense nationale, à Ottawa, le 2 février 1942, le message suivant:

La formation immédiate du $4^{\mathrm{e}}$ Régiment canadien d'artillerie moyenne est autorisée par la présente... Ce Régiment doit être prêt à traverser outre-mer aussitôt que possible après le $1^{\text {er }}$ mai [1942]. Tout sont personnel devra être de langue française ${ }^{3}$.

Une fois le $4^{\circ}$ Régiment officiellement constitué, il fallut lui assurer un esprit de corps essentiel à tout groupe d'hommes destinés à risquer leur vie ensemble.

Le 12 mars 1942, il était définitivement groupé à Petawawa, pour s'y entraîner jusqu'à son départ pour l'Angleterre, qui eut lieu le 8 août suivant. Son séjour en Angleterre dura du 18 août au 9 juillet 1944, soit environ deux ans, au cours desquels il devint une des unités d'artillerie les mieux entraînées de l'armée canadienne.

2a Le major Gagnon, aujourd'hui brigadier et ingénieur de profession, succéda au lieut.-col. Panet en Angleterre, celui-ci ayant dû se démettre de ses fonctions pour cause de maladie. Le brigadier Gagnon commanda le Régiment avec un succès éclatant, pendant toute la campagne du nordouest de l'Europe. C'est en grande partie grâce aux archives personnelles du brigadier Gagnon, gracieusement mises à la disposition de l'auteur, que ce récit a pu être reconstitué.

${ }^{2 b}$ Le major Archer, aujourd'hui brigadier et ingénieur de profession, était, jusqu'à il y a quelques mois, président du Conseil des ports nationaux à Ottawa.

3 Journal de guerre du Régiment, I: 1 (traduit de l'anglais par l'auteur); original déposé aux archives du ministère de la Défense nationale, à Ottawa. 


\section{BATAILLE DE NORMANDIE}

\section{AUTOUR DE CAEN}

Débarqué à Graye-sur-Mer, près de Courseulles, le matin du 9 juillet 1944, le Régiment était aussitôt dirigé vers une zone de concentration située à un kilomètre au sud-ouest de Ste-Croixsur-Mer. Le soir même, une vague de bombardiers allemands venaient pilonner ses positions.

$\mathrm{Du}$ côté allié, même si l'opération «Overlord» avait été préparée avec minutie pendant de longs mois, au cours desquels l'armée canadienne était devenue, au dire de son historien officiel, « une force exceptionnellement bien entraînée » ${ }^{4}$, il n'y avait pas lieu, comme on vient de le voir, de sous-estimer trop vite l'âpreté de la défense allemande. A cet égard, le témoignage du lieutenant-général Foulkes, qui était alors commandant de la $2^{\text {e }}$ division canadienne d'infanterie, est révélateur et capital, d'autant plus qu'il souligne, entre autres éléments du succès de nos armes, l'importance primordiale de l'artillerie dans la défaite de la Wehrmacht en Normandie:

Quand nous avons commencé à nous battre... à Caen, nous nous sommes heurtés à des troupes allemandes ayant l'expérience de la bataille et nous nous sommes rendu compte que nous n'étions pas de taille à nous mesurer avec elles. Nous n'aurions pas réussi sans l'appui de notre artillerie et de notre aviation ${ }^{5}$.

Récapitulons ce qui s'était passé du 6 juin au 9 juillet 1944, c'est-à-dire depuis l'ouverture du second front jusqu'au débarquement du Régiment en Normandie.

Dès le 10 juin, Rommel écrivait à Hitler: «Nos soldats des diverses armes combattent avec un acharnement et une opiniâ-

4 Ministère de la Défense nationale, L'Armée canadienne à la guerre: les Canadiens en Grande-Bretagne (1939-1944) (Ottawa, Imprimerie de Sa Très Excellente Majesté le Roi, 1946), 79.

5 Colonel C. P. Stacey, Histoire officielle de l'Armée canadienne pendant la seconde guerre mondiale: Six Années de Guerre: l'Armée au Canada, en Grande-Bretagne et dans le Pacifique (Ottawa, Imprimeur de la Reine, 1957), 262. 
treté extraordinaires ${ }^{6}$, face aux 640 pièces d'artillerie de la marine alliée. Le 17 juin, afin de pouvoir étudier la situation sur les lieux mêmes du combat, Hitler se rendait à Soissons pour conférer avec Rundstedt et Rommel. Faisant mine d'être impatient de se rendre sur le front de Caen en personne, Hitler jugeait toutefois à propos de déguerpir de Normandie dès le lendemain, après qu'un V-1 (bombe volante) eût failli le réduire en pièces dans son poste de commandement ?

A la fin de juin, alors que sept et demie des huit divisions Panzer de Rommel étaient employées à contenir les assauts de la $2^{\mathrm{e}}$ armée britannique autour de Caen ${ }^{8}$, « les deux artilleries ne cessaient de se contre-battre $»$, rapporte un témoin normand ${ }^{9}$. Le 4 juillet, « un violent tir d'artillerie se déclenche sur nous » raconte un autre témoin oculaire de la bataille de Caen ${ }^{10}$. C'était en effet ce jour-là que Carpiquet, aérodrome de la banlieue de Caen, était attaqué par la $3^{\mathrm{e}}$ division canadienne ${ }^{11}$.

\section{CHUTE DE CAEN}

Il s'agissait du prélude à l'attaque décisive contre Caen. En effet, le 8 juillet, la $3^{\mathrm{e}}$ division canadienne, les $3^{\mathrm{e}}$ et $59^{\mathrm{e}}$ divisions britanniques et deux brigades de chars, appuyées par 2,300 tonnes de bombes larguées par la R.A.F., se lançaient à l'attaque de la ville ${ }^{12}$.

Le 9 juillet, Caen tombait aux mains des troupes anglocanadiennes, bien que Rommel, disposant toujours de ses huit

${ }^{6}$ Maréchal Erwin Rommel, La Guerre sans haine, carnets présentés par le capitaine Liddel Hart, II: Les Années de défaite, traduction de Jacques Mordal, Collin Delavaud, Henri Daussy et René Jouan (Paris, Amiot-Dumont, 1953), 262.

7 Ibid., 263-4.

8 Chester Wilmot, The Struggle for E'urope (Londres, Collins, 1952), 336-7-8.

9 René Herval, Bataille de Normandie, récits de témoins recueillis et présentés par René Herval (Paris, Notre Temps, 1947), I: 382.

10 Ibid., 253.

11 John North, North-West Europe (1944-45): the Achievement of 21st Army Group (Londres, Her Majesty's Stationery Office, 1953), 55. 12 Ibid. 
divisions Panzer, interdît encore le passage de l'Orne, où la bataille était devenue une formidable mêlée de matériel ${ }^{13}$.

Le 10 juillet, alors que le Régiment était toujours concentré à Ste-Croix-sur-Mer, attendant l'ordre d'entrer dans la mêlée, un témoin normand notait que «depuis trois heures la bataille a repris du côté de Caen et de la route de Falaise ${ }^{14}$. De fait, à cette date où le Régiment s'apprêtait à entrer dans la bataille, la situation en Normandie avait atteint un point critique: la tentative de percée américaine, du côté de la Bretagne, avait échoué, et la route de Falaise restait toujours bloquée ${ }^{15}$.

\section{PREMIÉRES POSITIONS DE COMBAT DU REGIMENT}

Le 11 juillet, alors qu'un jeune SS rendait un hommage assez révélateur à l'artillerie alliée en avouant à un Français: «... de l'artillerie, ils en ont plein le c...» ${ }^{16}$, le brigadier $\mathrm{E}$. R. Suttie, commandant du $2^{\mathrm{e}}$ groupe canadien d'artillerie, convoquait une première conférence des commandants d'artillerie moyenne en Normandie. A la suite de cette conférence, dans l'après-midi, le groupe de reconnaissance du Régiment se dirigeait vers sa première position de combat, près du village de Rots, où «une odeur nauséabonde de chair corrompue empeste l'atmosphère » ${ }^{17}$, et où, quelques jours auparavant, un mariage s'était déjà célébré entre une jeune Normande et un sergent canadien-français. Voici comment un témoin de l'époque rapporte cet incident, plutôt significatif de la tactique amoureuse expéditive des Canadiens français :

Les Canadiens furent les bienvenus et, la plupart Canadiens francais engagés volontaires comme leurs pères en 1914, ils sympathisèrent tout de suite avec la population quelque peu surprise par leur parler nuancé d'un accent normand ... Dans les campagnes l'accueil ne dut pas moins être chaleureux car à

13 Général L.-M. Chassin, Histoire militaire de la seconde guerre mondiale (Paris, Payot, 1951), 275.

14 Herval, op. cit., 275.

15 Wilmot, op. cit., 311.

16 Herval, op. cit., 264.

17 Pierre Sévigny, Face à l'ennemi (Montréal, Beauchemin, 1946), 22. 
Rots, par exemple, fut célébré le mariage d'une jeune Normande avec un sergent de l'armée canadienne ${ }^{18}$.

$\mathrm{Si}$, comme on vient de le voir, l'accueil des Français était chaleureux, la topographie des environs de Rots était beaucoup moins invitante. En effet, la région était encore tout infestée de mines allemandes, de sorte que la reconnaissance de la position dut se faire à l'aide de détecteurs de mines. Bien qu'aucune de celles-ci n'éclatât, la Luftwaffe, pour sa part, commença aussitôt à exercer ses ravages sur la position qu'essayait de préparer l'avant-garde du Régiment. Trois avions de chasse allemands furent abattus ce jour-là au-dessus de cette position.

Malgré cette manifestation brutale de la résistance allemande à l'avance du Régiment en Normandie, le 12 juillet, à 11 heures du soir, le gros du Régiment quittait Ste-Croix-sur-Mer pour se diriger, en pleine obscurité, -_ zébrée seulement par les éclairs intermittents de l'artillerie déjà en place, - vers sa première position de combat ${ }^{19}$.

De nouveau, la Luftwaffe fit son apparition pour paralyser bientôt l'avance du Régiment sur la route découverte entre Ste-Croix et Rots. Chacun se blottit aussitôt à l'abri des véhicules. Le spectacle que présentait alors cette nuit de juillet 1944 en terre normande est inoubliable. Voici comment le capitaine Sévigny ${ }^{19 a}$ devait plus tard évoquer cette scène, dans ses souvenirs de guerre:

La nuit brille de toutes ses étoiles. Je me trouve dans un véhicule ouvert et ne me lasse pas d'admirer le spectacle offert à mes yeux. Les flammes des villages en feu montent à l'horizon; notre aviation attaque des objectifs ennemis; je vois éclater des bombes; les obus des canons antiaériens allemands strient le ciel d'éclairs. Sur la route, j'aperçois, à perte de vue, dans la clarté de cette fantasmagorie, l'interminable et mouvante perspective des voitures

18 Herval, op. cit., 264.

19 Journal de guerre du Régiment, XXVIII: 5.

19a Aujourd'hui lieutenant-colonel et ministre associé de la Défense nationale du Canada. 
blindées de toute une armée montant à l'assaut. Derrière nous, à intervalles réguliers, les salves des canons de marine illuminent d'une lueur fulgurante le paysage environnant. L'hallucination de ce feu d'artifice, le vacarme infernal des détonations, le roulement de notre caravane d'acier sur des routes d'asphalte: c'est le feu de l'action ${ }^{20}$.

C'était en effet le feu de l'action dans toute sa terrifiante beauté. Deux Spitfires, qui se trouvaient dans la mêlée au-dessus du convoi, furent abattus. On a prétendu que le Régiment, dans son zèle de néophyte, en abattit un troisième, par la main du sergent-major Gérald Trottier, dont les doigts frétillaient toujours fiévreusement autour de la gâchette de sa Bren, au moindre indice de vrombissement. Le Spitfire soi-disant atteint par le feu du sergent-major Trottier s'abattit tout près de la troupe $\mathrm{D}$ du Régiment, et le malheureux pilote anglais fut tué. Ce fut là un de ces incidents regrettables que l'aviation britannique et américaine devait compenser bientôt au centuple, au grand détriment du Régiment, comme nous le verrons plus loin. Mais c'était là un de ces impondérables du combat qu'il était impossible d'éviter dans une mêlée aussi confuse.

Le 13 juillet, à 5 heures du matin, le Régiment était prêt à tirer sur l'ennemi. Ce dernier avait déjà commencé à goûter à la « furia » canadienne-française, si l'on en juge par cet extrait de lettre d'un officier du Régiment, datée de ce même jour:

J'ai entendu raconter de la bouche de Français la façon extraordinaire dont nos troupes canadiennesfrançaises d'assaut ont donné la frousse aux Boches. Il paraît que c'en était comique de les voir courir, quand ils ont vu nos gaillards, armés du couteau: les talons leur touchaient aux fesses ${ }^{21}$.

C'était maintenant au tour de l'artillerie canadienne-française à suivre les traces de ses vaillants prédécesseurs de l'infanterie: les gars du Chaudière, du Maisonneuve et du Fusiliers Mont-Royal. En effet, dans une autre lettre, datée du même jour, ce même officier écrivait: « Nous tirons sur les Boches...

20 Sévigny, op. cit., 21-2.

21 Archives de l'auteur, [Rots], 13 juillet 1944. 
avec succès, et sans réponse. Ils n'osent pas répondre, car on les réduirait en cendres ${ }^{22}$.

Une fois le Régiment en batterie autour de Rots, le capitaine Claude Chabot avait établi un poste d'observation dans SaintGermain-la-Blanche-Herbe, face au bocage de Louvigny, aux abords de Caen. Dès 11 h. 40, le matin du 13 juillet, le capitaine Chabot avait donné son premier ordre de feu à sa troupe D, où le sergent Plouffe avait l'honneur d'être le premier canonnier du $2^{\text {e }}$ groupe canadien d'artillerie à tirer sur les Allemands ${ }^{23}$.

Le tir se poursuivit tout l'après-midi du 13 juillet. Près de leurs pièces, les canonniers du Régiment avaient déjà pu se creuser des abris de fortune et manifester ainsi leur merveilleux esprit d'initiative et d'invention, dont ils avaient grand besoin d'ailleurs, car l'artillerie ennemie ne tardait pas à les engager. De fait, les Allemands ne mirent guère de temps à repérer le poste d'observation du capitaine Chabot, auprès de qui s'était rendu dans l'intervalle le major Codère, commandant de la $58^{\circ}$ batterie. Bientôt ces deux officiers étaient blessés et évacués à l'arrière. Le commandant, - le lieut.-col. Réal Gagnon, qui avait succédé au lieut.-col. Panet, en Angleterre, - décida alors sans délai d'établir un nouveau poste, cette fois destiné au capitaine Sévigny. Celui-ci s'était déjà familiarisé avec les objectifs qu'il devait bientôt engager, en grimpant sur la tour de l'ancienne abbaye d'Ardennes, près de Cussy, où quelques jours plus tôt le général Kurt Meyer avait fait exécuter des prisonniers canadiens.

\section{PUISSANCE ET PRÉCISION DE L'ARTILLERIE ALLIÉE}

A cette date précisément où le Régiment commençait à tirer ses premiers obus sur l'ennemi, il est intéressant de noter ce témoignage révélateur d'un civil normand: «Nous étions habitués à recevoir des obus, mais vraiment cette nuit les limites permises ont été dépassées ${ }^{24}$.» Il faut dire que les Allemands, de leur

22 Ibid.

23 Journal de guerre du Régiment, XXVIII: 6 .

24 Herval, op. cit., 255. 
côté, n'étaient pas inactifs. En effet, si Caen était maintenant occupée par nos troupes avancées, la résistance ennemie tenait toujours dans la banlieue, - dans le faubourg de Vaucelles plus précisément, - au sud de l'Orne. Aussi, la Luftwaffe venait-elle de nouveau harceler les positions du Régiment le 15 et le 16 juillet. Mais, malgré les effets parfois paralysants de l'aviation et de l'artillerie ennemies, l'infanterie alliée rapportait que le feu du Régiment était « rapide et précis », sous les ordres du capitaine Jean Mercier qui avait, entre-temps, remplacé le capitaine Sévigny au poste d'observation. Plusieurs cibles avaient en effet été engagées avec succès, notamment des positions d'artillerie ennemies. Voici comment, le 16 juillet, un officier du Régiment résumait la situation:

\begin{abstract}
... Aujourd'hui, il fait une chaleur torride comme à Montréal en été; nos canons ne cessent de harceler l'ennemi nuit et jour; les premières nuits, je n'ai pu dormir, mais maintenant je m'habitue au bruit... D'après les deux jeunes prisonniers que j'ai vus hier, les Allemands ne sont pas à craindre. En effet, dans l'après-midi d'hier, deux agents de la police militaire nous amenaient deux jeunes freluquets de dix-huit ans dont l'un était blessé à la jambe. Ils étaient cachés depuis cinq jours et attendaient le moment propice pour se rendre; ils faisaient pitié à voir; ils avaient l'air triste, abattu et résigné..${ }^{25}$
\end{abstract}

\title{
DÉGAGEMENT DE LA BANLIEUE SUD DE CAEN
}

Le lendemain, 17 juillet, ordre était donné aux $3^{\mathrm{e}}$ et $4^{\mathrm{e}}$ régiments d'artillerie moyenne de se préparer à appuyer la $3^{\mathbf{e}}$ division canadienne dans une nouvelle tentative pour bouter définitivement les Allemands hors de la banlieue sud de Caen. Sept cent soixante canons de tous calibres devaient participer à cet assaut, appuyés par 10,000 tonnes de bombes promises par la R.A.F. ${ }^{26}$. Les plans de tir arrivaient au Régiment à 10 heures du soir, et le commandant se rapportait aussitôt au Q.G. de la $4^{\mathrm{e}}$ brigade canadienne pour coordonner le feu du Régiment. L'attaque de

25 Archives de l'auteur, [Rots], 16 juillet 1944.

26 Journal de guerre du Régiment, XXV: 5. 
la $3^{\text {e }}$ division était prévue pour l'aube du 18 juillet, et son objectif était tout le terrain situé au nord de l'Orne, face à Louvigny.

Trois jours plus tôt, soit le 15 juillet, Rommel avait écrit ce qui suit au haut commandement allemand à Berlin :

... Sur le front de Normandie, la situation ne cesse d'empirer et le dénouement est proche. Etant donné l'acharnement des combats, la dépense considérable de matériel à laquelle se livre l'ennemi ... surtout pour l'artillerie et les blindés... la $7^{\mathrm{e}}$ armée a un urgent besoin de deux divisions fraîches, les troupes qui combattent en Normandie étant maintenant à bout de forces ${ }^{27}$.

Les Allemands étaient-ils à ce point épuisés ? Pour peu qu'on connaisse la furie des combats qui devaient se dérouler par la suite, de Caen jusqu'à Falaise, on serait porté à croire que Rommel écrivait alors avec le vague pressentiment que sa blessure du 17 juillet allait le mettre définitivement hors de combat.

Mais la blessure de Rommel était également un incident regrettable pour les alliés occidentaux, puisqu'on prétend maintenant que s'il n'eût pas été blessé le 17 juillet, dès la nouvelle de l'attentat contre Hitler, trois jours plus tard, il aurait mis bas les armes devant les alliés occidentaux ${ }^{28}$.

$\mathrm{Au}$ prix donc de nombreuses autres vies humaines, inutilement sacrifiées, le combat se poursuivit avec le même acharnement après le départ de Rommel, sous la direction du maréchal von Kluge, vétéran aguerri du terrible front russe, qui assuma dès le 17 juillet à la fois les fonctions de Rommel et de von Rundstedt, celui-ci ayant été limogé entre-temps.

On se battait donc encore avec furie le 18 juillet dans le faubourg de Vaucelles, alors qu'une température idéale favorisait en tous points la supériorité aérienne des alliés, comme en fait foi ce témoignage:

\footnotetext{
27 Rommel, op. cit., 273.
}

28 Ibid. 
Dans le ciel les bombardiers passent... Les vagues se succèdent sans interruption. Ils vont par groupes de six, de vingt-quatre, de cinquante et lấchent leurs torpilles inlassablement... 8,000 tonnes de bombes ont été déversées de l'autre côté de l'Orne. Vaucelles sera dégagé ${ }^{29}$.

Dans la nuit de ce même jour, le Régiment, de son côté, poursuivait son avance vers sa deuxième position de combat, près de Cussy encore fumant sous les ruines, où il devait appuyer une attaque de la $5^{\mathrm{e}}$ brigade canadienne d'infanterie dans l'aprèsmidi. Les environs de Cussy, dont les hameaux de Buron, d'Authie et de Gruchy, présentaient alors une scène d'affreuse désolation. Voici en quels termes le capitaine Sévigny devait plus tard évoquer ce tableau dans ses souvenirs:

... Tout était détruit ou endommagé; dans les champs, dans les cours de ferme, d'innombrables cadavres d'animaux se décomposaient. A l'intérieur des maisons farouchement défendues par les Allemands, des soldats morts l'arme à la main gisaient dans les positions les plus étonnantes ... Au creux d'un fossé, je vis, enlacés dans la mort, le cadavre d'un parachutiste allemand et celui d'un commando britannique: à la même seconde, ils s'étaient poignardés d'un même tragique élan. L'Anglais, la main crispée sur le manche de son poignard, tenait celuici enfoncé jusqu'à la garde dans la gorge du Boche; les doigts raidis du Boche serraient un couteau plongé jusqu'au manche dans le dos de son agresseur. Boche et Anglais se regardaient et leurs visages, figés de haine et de rage, conservaient dans la mort une expression terrifiante ${ }^{30}$.

Dès que le Régiment fut en batterie près de Cussy, le 19, on réclama son feu meurtrier, à la fois des postes d'observation régimentaires établis autour de Caen et d'un poste d'observation aérien, vu que les Allemands contre-attaquaient sans cesse. De fait, même après la libération de Caen, la ville restait encore soumise à un feu nourri de la part de l'artillerie allemande, ce qui empêcha, au dire d'un témoin normand, le déroulement

29 Herval, op. cit., 258.

30 Sévigny, 24-5. 
traditionnel des fêtes du 14 juillet. D'où la nécessité pour l'artillerie alliée d'exercer un tir de contrebatterie incessant; et cela, même si on ne craignait plus le retour offensif des Allemands, qui devaient maintenant se trouver à six kilomètres de la ville, en direction de Falaise.

Le $2^{\mathrm{e}}$ corps canadien, qui projetait alors une nouvelle attaque destinée à déloger définitivement les Allemands de la rive sud de l'Orne, comptait sur l'appui de l'artillerie moyenne pour réussir. En effet, des ordres arrivaient au Régiment le soir même du 19, et les préparatifs se poursuivaient dès le lendemain, 20 juillet, date fatidique où devait échouer en Allemagne le dernier attentat contre la vie de Hitler. Deux officiers observateurs, les capitaines Mercier et Sévigny, ainsi que deux officiers représentant le Régiment, le major Édouard Tremblay ${ }^{30 a}$ et le capitaine Marcellin Lahaie ${ }^{30 b}$, se rapportaient tôt le matin du 20 au Q.G. de la $2^{\text {e }}$ brigade canadienne de chars ${ }^{31}$. Ce jour-là, un officier du Régiment écrivait à sa femme:

... La machine militaire allemande se désagrège rapidement et notre victoire est certaine... Quelques minutes de loisirs m'ont permis d'errer à travers une ancienne abbaye à moitié détruite par les bombes... Apparemment, les Boches ont fui en vitesse, car c'est encore plein de butin; la table est encore servie et l'on peut voir des morceaux de pain moisi qui traînent dans les assiettes... Les nouvelles furent excellentes cet après-midi, et nous continuons de harceler l'ennemi sans relâche... La fin approche ${ }^{32}$.

Pendant que se préparait cette attaque, le groupe de reconnaissance du Régiment était déjà parti de Cussy pour aménager, parmi les décombres du faubourg de Vaucelles, libéré depuis trente-six heures seulement, une nouvelle position du combat ${ }^{33}$.

30a Aujourd'hui lieutenant-colonel et architecte de profession.

30b Aujourd'hui lieutenant-colonel et attaché militaire du Canada en France, après avoir été le premier directeur du Collège Militaire Royal de Saint-Jean.

31 Journal de guerre du Régiment, XXVIII : 9.

32 Archives de l'auteur, [Cussy], 20 juillet 1944.

33 Journal de guerre du Régiment, XXVIIII : 9. 
Le gros du Régiment s'y rendit pendant la nuit, alors que des difficultés de cartographie égarèrent quelques membres intrépides du Régiment dans les lignes avancées de l'infanterie, situation peu familière à des artilleurs ${ }^{34}$.

Le 21 juillet, à $4 \mathrm{~h}$. du matin, le Régiment était en batterie dans un vallon découvert, immédiatement au sud du faubourg de Vaucelles. Il devait subir là, pendant environ trois semaines, sans interruption, les attaques violentes de l'artillerie et de l'aviation allemandes et, immédiatement avant son départ de ce vallon de la mort, par suite d'une erreur, un bombardement désastreux de la part de l'aviation américaine. Ce sera un baptême de feu sanglant et inoubliable.

$$
\text { (à suivre) }
$$

JACQUES GOUIN, ex-lieutenant d'artillerie, diplômé en sciences politiques (Ottawa), correspondant canadien à la Revue d'Histoire de la $2^{\mathrm{e}}$ guerre mondiale (France).

34 Ibid. 